\title{
Prospects for Advanced Engineering Design Based on Risk Assessment
}

\author{
M. Holický
}

Current approaches to the design of structures are based on the concept of target probability of failure. This value is, however, often specified on the basis of comparative studies and past experience only. Moreover, the traditional probabilistic approach cannot properly consider gross errors and accidental situations, both of which are becoming more frequent causes of failure. This paper shows that it is useful to supplement a probabilistic design procedure by a risk analysis and assessment, which can take into account the consequences of all unfavourable events. It is anticipated that in the near future advanced engineering design will include criteria of acceptable risks in addition to the traditional probabilistic conditions.

Keywords: reliability, hazard situations, adverse events, costs, risk assessment, Bayessian network, advanced engineering design.

\section{Notation}

$\mathrm{g}(\boldsymbol{x})$

$C_{i j}$

$C_{\text {tot }}$

$E_{i j}$

$H_{i}$

$H_{1}$

$\mathrm{H}_{2}$

$\mathrm{P}\left(F \mid H_{i}\right) \quad$ Probability of failure $\mathrm{F}$ given the situation $H_{i}$

$p_{F} \quad$ Probability of failure $F$

$p_{d} \quad$ Target probability of failure

$p_{f} \quad$ Probability $\mathrm{P}\left(F \mid H_{2}\right)$ of structural failure during fire

$p_{f, s} \quad$ Probability of fire start $\mathrm{P}\left(\mathrm{H}_{2}\right)$

$p_{f i, d} \quad$ Conditional probabilities of fire flashover given $\mathrm{H}_{2}$

$p_{f i} \quad$ Probability of fire flashover

$p_{t, f i} \quad$ Target probability of structural failure under fire design situation

$x \quad$ Generic point of the vector of basic variables.

$\boldsymbol{X} \quad$ Vector of basic variables

$\beta \quad$ Reliability index

$\varphi_{\boldsymbol{X}}(\boldsymbol{x}) \quad$ Probability density function of the vector of basic variables $\boldsymbol{X}$

$\Phi_{X}(\boldsymbol{x}) \quad$ Distribution function of the vector of basic variables $\boldsymbol{X}$

\section{Introduction}

Design and assessment of civil structures suffer from a number of uncertainties, which can hardly be described by available theoretical tools. According to Thoft-Christensen and Baker [1], Melchers [2] and Holický [3] these uncertainties include:

- natural randomness of basic variables,

- statistical uncertainties caused by a limited size of available data,

- model uncertainties caused by deficiencies of computational models,

- uncertainties caused by inaccuracy in definitions of limit states,

- gross errors caused by human faults,
- lack of understanding of actual behaviour of materials and structures.

These uncertainties are listed in the order corresponding to their increasing effect on the frequency of failures and the decreasing possibility of describing them theoretically. Traditional probability methods usually deal with the first three types of uncertainties only. It was shown by Holický [3] that the fourth type of uncertainty can be partly described using the theory of fuzzy sets. Theoretical tools for the description of gross errors are insufficient (as indicated by Melchers [2]), while no tools are available to describe the lack of understanding of the actual behaviour of new materials and structures. The available theoretical tools obviously have a limited capability of describing all types of uncertainties. This adverse reality corresponds to the observed proportions of failure causes, for which informative values are indicated in Table 1 (obtained from the data provided by Melchers [2], Stewart and Melchers [4] and other publications quoted in these references).

The first line in Table 1 indicates the proportions of various origins of structural failures chosen from basic activities during the construction and service-life of structures. The second line indicates relations between these activities and two main causes: gross errors (about $80 \%$ ) due to human activity and environmental effects (about $20 \%$ ), which are not directly dependent on human activity.

Table 1: The proportions of causes of structural failures

\begin{tabular}{|c|cccc|}
\hline \multirow{2}{*}{ Origin } & Design & Execution & Use & Other \\
\cline { 2 - 5 } & $20 \%$ & $50 \%$ & $15 \%$ & $15 \%$ \\
\hline \multirow{2}{*}{ Causes } & $\begin{array}{c}\text { Gross errors due } \\
\text { to human activity }\end{array}$ & Environmental effects \\
\cline { 2 - 4 } & \multicolumn{2}{|c|}{$80 \%$} & $20 \%$ & \\
\hline
\end{tabular}

Environmental influences include both random and hazard (accidental) situations, e.g. due to impact, explosion, fire and extreme climatic actions. Thus, natural randomness causes only a small proportion of the failures (about $10 \%$ ). Obviously, further development of more precise procedures based on the traditional probabilistic approach (the basis of which is mentioned in the following section) has only a limited significance. Advanced engineering design methods 
should therefore attempt to consider the actual causes of failures.

\section{The probabilistic method}

The probabilistic method of designing structures assumes that a failure $F$ of the structure is unequivocally described by inequality $\mathrm{g}(x)<0$, where $\mathrm{g}(x)$ denotes the limit state function $(\mathrm{g}(x)=0$ describes the limit state, $\mathrm{g}(x)>0$ the safe state), $\boldsymbol{x}$ is a realisation of the vector of basic variables $\boldsymbol{X}$. If $\varphi_{\boldsymbol{X}}(\boldsymbol{x})$ indicates the joint probability density of the vector of the basic variables $\boldsymbol{X}$, the probability of failure $p_{F}$ can be determined from the relation

$$
p_{F}=\int_{g(x)<0} \varphi_{\boldsymbol{X}}(\boldsymbol{x}) \mathrm{d} \boldsymbol{x} .
$$

The reliability index $\beta$ is formally defined on the basis of the probability $p_{F}$ using the relation $p_{F}=\Phi(-\beta)$, where $\Phi$ is the distribution function of the standardised normal distribution. Calculation of the probability of failure $p_{F}$ using equation (1) suffers from two essential deficiencies, as demonstrated by Ellingwood [5]:

- uncertainty in the definition of the limit state function $g(x)$,

- uncertainties in theoretical models describing the basic variables $\boldsymbol{X}$.

These deficiencies are most likely the main sources of the observed discrepancy between the determined probability $p_{F}$ and the actual frequency of failures. That is why quantities $p_{F}$ and $\beta$ are often referred to as „formal“ (notional) reliability indicators (associated with the intention to standardise theoretical models of basic variables). However, such an approach jeopardises the nature of probabilistic concepts including the methods of probabilistic optimisation, which should provide the target probability of failure $p_{d}$ used in the design condition $p_{F}<p_{d}$. In order to increase the significance of probabilistic concepts a considerable effort focussed on improving the theoretical models describing basic variables and on extending of the traditional probabilistic concepts by risk assessment methods has recently been observed by Stewart and Melchers [4] and Ellingwood [5].

\section{The concept of acceptable risk}

The risk assessment of a system attempts to cover all possible events that might lead to unfavourable effects related to the considered system. As mentioned above, these events are caused mainly by gross errors in human activity and by accidental actions such as impact, explosion, fire and extreme climatic loads. Adequate situations (hazard scenarios and common design situations), designated generally as $H_{i}$, will occur with the probability $\mathrm{P}\left(H_{i}\right)$. If the failure of structure $F$ due to a particular situation $H_{i}$ occurs with the conditional probability $\mathrm{P}\left(F \mid H_{i}\right)$, then the total probability of failure $p_{F}$ is given as:

$$
p_{F}=\sum_{i} \mathrm{P}\left(F \mid H_{i}\right) \mathrm{P}\left(H_{i}\right) .
$$

The conditional probabilities $\mathrm{P}\left(F \mid H_{i}\right)$ must be determined by a separate analysis of the respective situations $H_{i}$. Equation (2) can be used for harmonisation of the partial probabilities of failure $\mathrm{P}\left(F \mid H_{i}\right) \mathrm{P}\left(H_{i}\right)$ corresponding to the situations $H_{i}$, and for the following risk consideration.

In general, the situations $H_{i}$ may cause a number of unfavourable events $E_{i j}$ (e.g. excessive deformations, full development of the fire). It is assumed that the adverse consequences of these events can be expressed by a one-component quantity $C_{i j}$ (for example, by the cost expressed in a certain currency). It is further assumed that the consequences $C_{i j}$ are mutually uniquely related to the events $E_{i j}$. Then the total risk $C$ related to the considered situations $H_{i}$ is the sum

$$
C=\sum_{i j} C_{i j} \mathrm{P}\left(E_{i j} \mid H_{i}\right) \mathrm{P}\left(H_{i}\right) .
$$

It is sometimes necessary to describe the consequences of an unfavourable phenomenon $E_{i j}$ by a quantity having several components, denoted as $C_{i j, k}$ (describing for example cost, injuries or casualties). The components $C_{k}$ of the resultant risk are then given as

$$
C_{k}=\sum_{i j} C_{i j, k} \mathrm{P}\left(E_{i j} \mid H_{i}\right) \mathrm{P}\left(H_{i}\right) .
$$

If it is possible to specify the acceptable limit $C_{k, d}$ for the components $C_{k}$, it is possible to design the structure on the basis of the condition of acceptable risks $C_{k}<C_{k, d}$ that supplements the probability condition $p_{f}<p_{d}$.

\section{Example of a structure under a fire situation}

An example illustrating the concept of acceptable risks concerns a structure for which only two different situations are considered:

- $H_{1} \quad$ persistent design situation, for which $\mathrm{P}\left(H_{1}\right)=0.99$ is assumed,

- $H_{2}$ accidental situation during the fire, for which $\mathrm{P}\left(H_{2}\right)=0.01$ is assumed.

The persistent situation $H_{1}$ is analysed using the traditional probabilistic reliability analysis. An example of an analysis of situation $\mathrm{H}_{2}$ is indicated in Figure 1, which shows a Bayesian network describing the structure during a fire.

The chance, decision and utility nodes indicated in Figure 1 are briefly described below. A more detailed description is given by Holický and Schleich [6]. An alternative type of network and analysis was recently provided by Holický and Schleich [7].

1 - Fire starts. The parentless chance node describing the initiation of a fire. The probability $p_{f i, s}=\mathrm{P}\left(H_{2}\right)=0.01$ is assumed for the positive state (fire starts) considering an office compartment of $25 \mathrm{~m}^{2}$ during its design life of 50 years.

2 - Detection by occupants. The chance node describing the detection of smoke by occupants or neighbours within a suitable time period. The conditional probability 0.9 given the fire started (parent node 1) is considered.

3 - Occupancy. The chance node describing the activity of the occupants of the building to diminish the fire. The conditional probabilities related to the states of parent nodes 2 and 6 are given by Holický and Schleich [6]. 


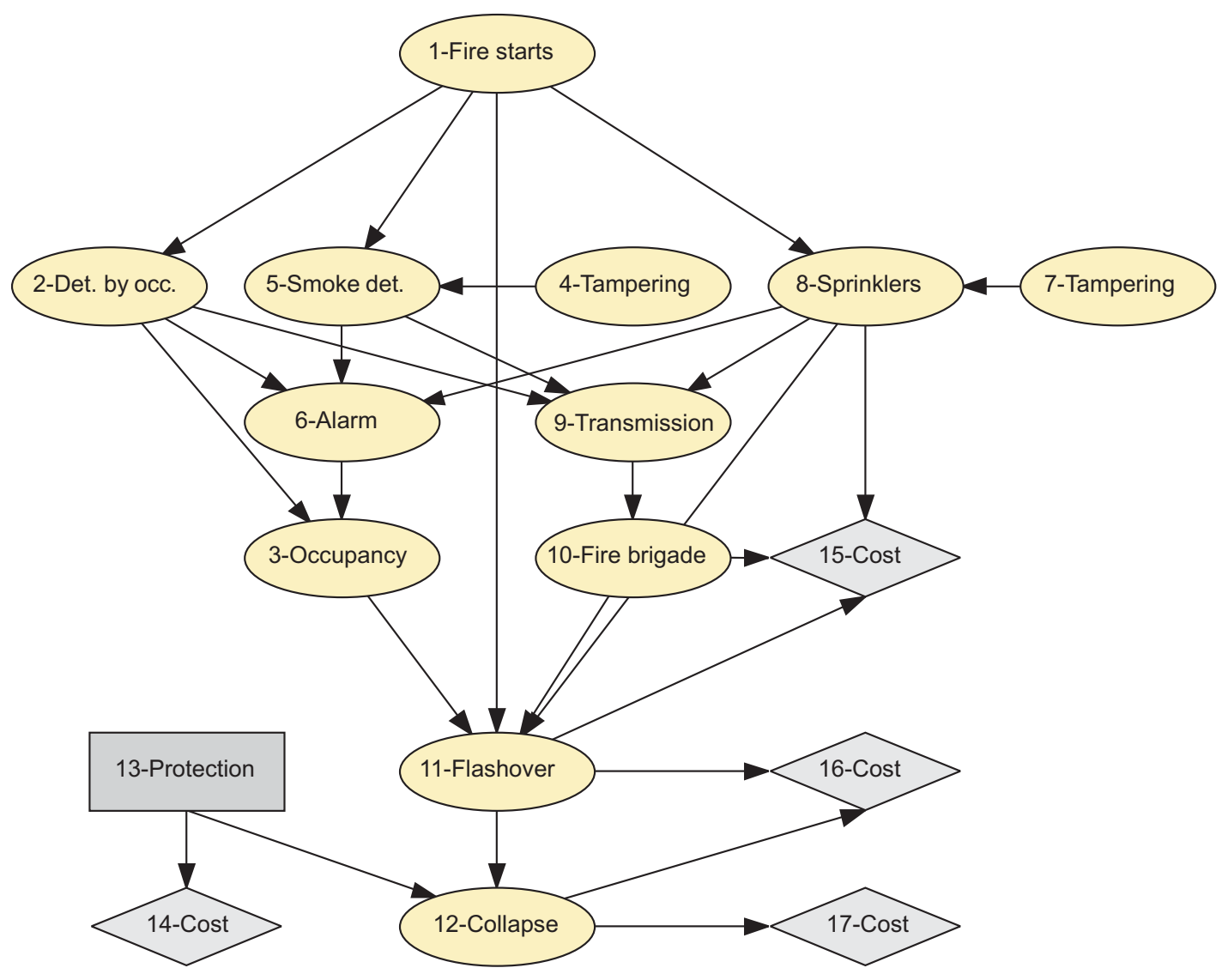

Fig. 1: An example of the Bayesian belief network representing a fire situation

4 - Tampering. This parentless chance node describes the interference of random factors with the automatic fire detection system (node 5). The probability 0.02 is considered for the disturbing effects on the detection system.

5 - Smoke detection. The chance node describing the operation of an automatic smoke detection system. The conditional probabilities related to parent nodes 1 and 4 are given by Holický and Schleich [6].

6 - Alarm. The chance node describing the operation of an acoustic fire alarm system. The conditional probabilities related to the states of parent nodes 2,5 and 8 are considered in accordance with Holický and Schleich [6].

7 - Tampering. The parentless chance node describing the interference of random factors with the automatic sprinkler system (node 5). The probability 0.02 is considered for the disturbing effects on the sprinkler system.

8 - Sprinklers. The chance node describing the operation of the automatic sprinkler system (if installed). The conditional probabilities related to the states of parent nodes 1 and 7 are indicated by Holický and Schleich [6].

9 - Transmission. The chance node describing the operation of manual or automatic alarm transmission to the fire brigade. The conditional probabilities related to the states of parent nodes 2, 5 and 8 are given by Holický and Schleich [6].
10 - Fire brigade. The chance node describing the operation of a professional fire brigade. The conditional probability 0.9 that the fire brigade is active when the alarm (parent node 9) goes off is considered.

11 - Flashover. The chance node describing the development of the fire. The conditional probabilities related to the states of parent nodes 1, 3, 8 and 10 are given by Holický and Schleich [6].

12 - Collapse. The chance node describing structural collapse under the fire design situation in the case of fire flashover. The conditional probability 0.2 of structural collapse given the fire flashover is considered in the example.

13 - Protection. The parentless decision node describing the resolution concerning protection of the structural against fire. The node has two states: 'yes' and 'no'. As indicated by Holický and Schleich[6] for the state "no" the child node 12-Collapse has greater probability of a positive state than for the positive decision "yes" concerning structural protection.

14 - Cost. The utility node describing the cost $C_{14}(13)$ of structural protection (affecting node 12), which depends on the state of node 13. The relative value 10 expressed in monetary units is considered if the decision (node 13) is positive. 
15 - Cost. The utility node describing the damage cost $C_{15}(8$, 10,11 ) caused by the sprinklers (node 8 ) and the fire brigade (node 10) if the fire (node 11) does not flashover. The relative costs expressed in the same monetary unit as cost $C_{14}(13)$ are indicated by Holický and Schleich [6]. If the fire develops fully (node 11), these costs are covered by utility node 16 .

16 - Cost. The utility node describing the damage cost $C_{16}(11,12)$ assuming that the fire flashover occurred. The relative value of 100 units expressed in the same monetary units as the costs $C_{14}(13)$ and $C_{15}(8,10,11)$ is assumed.

17 - Cost. The utility node describing the damage cost $C_{17}(12)$ due to the collapse of the structure (node 12). Relative values of the cost $C_{17}(12)$ from $10^{5}$ to $10^{8}$ are considered in the example.

Assuming the independence of the two situations $H_{1}$ and $H_{2}$, it holds that $\mathrm{P}\left(H_{1}\right)+\mathrm{P}\left(H_{2}\right)=1$. If $\mathrm{P}\left(F \mid H_{1}\right)=10^{-5}$ (which is an expected value) and $\mathrm{P}\left(F \mid H_{2}\right)=10^{-3}$, then the probability of failure is according to relation (2)

$$
p_{F}=\mathrm{P}\left(F \mid H_{1}\right) \mathrm{P}\left(H_{1}\right)+\mathrm{P}\left(F \mid H_{2}\right) \mathrm{P}\left(H_{2}\right) \approx 2 \times 10^{-5} .
$$

Further it is assumed that the following events, the first of which are related to situation $H_{1}$, and the other to $H_{2}$, may occur:

- $E_{11}$ structural failure due to exceeding the ultimate limit state,

- $E_{12}$ unacceptable deformations, i.e. exceeding the limit state of serviceability,

- $E_{21}$ activation of sprinklers (Figure 1 - chance node 8),

- $E_{22}$ intervention of a fire brigade (Figure 1 - chance node 10),

- $E_{23}$ full development of fire (Figure 1 - chance node 11),

- $E_{24}$ structural failure due to fire (Figure 1 - chance node 12).

The conditional probabilities $\mathrm{P}\left(E_{i j} \mid H_{i}\right)$ can generally be determined on the basis of a detailed probabilistic analysis of the two situations $H_{1}$ and $H_{2}$.

Assuming that unfavourable consequences are given by quantities $C_{i j}$ or $C_{i j, k}$ corresponding to an unfavourable phenomenon $E_{i j}$, equations (3) and (4) may be applied to determine the total risk $C$ or its components $C_{k}$. Note that the unfavourable consequences $C_{21}, C_{22}, C_{23}$ and $C_{24}$ are described in Figure 1 by utility nodes 15,16 and 17 . The total expected cost $C_{t o t}$ can then be given by a simplified equation (3) as a sum

$C_{t o t}=C_{14}(13)+C_{15}(8,10,11)+C_{16}(11,12)+p_{f} \times C_{17}(12)(6)$

where, as described above, $C_{14}(13)$ is the cost depending on the state of node $13, C_{15}(8,10,11)$ is the damage cost depending on the states of nodes 8,10 and 11 , and $C_{16}(11,12)$ is the cost due to flashover depending on the state of nodes 11 and 12. The last term in the sum $p_{f} \times C_{17}(12)$ is the expected cost due to structural failure (collapse), where $p_{f}$ is the probability of failure and $C_{17}(12)$ is the damage cost given the failure. The damage cost $C_{17}(12)$ is a complex quantity, which is dependent on many factors including the cost of the structure and other costs due to structural malfunctioning.

\section{Probabilistic analysis}

The Bayesian network was analysed using the program HUGIN 1999. The resulting probabilities $p_{f i}$ of fire flashover, the conditional probabilities $p_{f, d}$, and the probabilities of structural failure $p_{f}$ are shown in Table 2.

Table 2: Probabilities of fire flashover $p_{f i}$ and conditional probabilities $p_{f i, d}$

\begin{tabular}{|lcc|}
\hline $\begin{array}{l}\text { Decision concerning } \\
\text { protection }\end{array}$ & Yes \\
\hline $\begin{array}{l}\text { Probabilities of fire flashover } p_{f i} \\
\text { assuming } \mathrm{P}\left(H_{2}\right)=0.01\end{array}$ & 0.00013 \\
$\begin{array}{l}\text { Conditional probabilities } p_{f i, d} \text { of } \\
\text { fire flashover given } H_{2}\end{array}$ & 0.013 \\
$\begin{array}{l}\text { Probability of structural failure } \\
\text { during the fire } p_{f}=\mathrm{P}\left(F \mid H_{2}\right)\end{array}$ & $1.0 \times 10^{-5}$ & $3.6 \times 10^{-5}$ \\
\hline
\end{tabular}

The probability $p_{f i}$ of fire flashover $(0.00013)$ obtained by the probabilistic analysis of the network seems to be relatively low. Note, however, that this value is valid for the fire start probability $p_{f i, s}=\mathrm{P}\left(H_{2}\right)=0.01$ (corresponding to a small compartment area $A=25 \mathrm{~m}^{2}$ and a 50 year time period), which is linearly dependent on compartment area $A$. Thus, the input probability $p_{f i, s}$ may be much greater than 0.01 . If, for example, the compartment area is ten times greater $\left(250 \mathrm{~m}^{2}\right)$, then $p_{f i, s}=0.1$ and the probabilities $p_{f i}$ will also be ten times greater than the values indicated in Table 2 . The conditional probability $p_{f i, d}$ that the fire, once started, will develop fully (shown in the second line of Table 2), is relatively low primarily due to the relatively high efficiency of the sprinklers considered by Holický and Schleich [6].

Table 2 also shows that the probability of structural failure may be decreased using the appropriate structural protection. However, the data given in Table 2 depend on input conditional probabilities, which should be determined on the basis of a detailed probabilistic analysis, taking into account the actual protection measures. Having the probability of fire flashover $p_{f}$, it is now possible to specify the target probabilities $p_{t, f_{i}}$ of structural failure under the fire design situation using equation (2). Obviously with increasing probability of fire flashover $p_{f i}$ the probability $p_{t, f i}$ decreases. As $p_{f i}$ is dependent on compartment area $A$, the probabilities $p_{t, f i}$ are also dependent on $A$. Detailed discussion is provided by Holický and Schleich [6].

For large compartment areas $A$, the target probability $p_{t, f i}$ of structural failure under the fire design situation will be very small and, consequently, it may be difficult (if not impossible) to design the structure under this condition. In such a case, it may be necessary to use additional elements of the fire protection system in order to decrease the probability of fire flashover $p_{f i}$. It appears that the Bayesian network may effectively be used to model a fire protection system and, possibly, to find the optimum arrangement. For this purpose decision and utility nodes often supplement a Bayesian network like that in Figure 1. 


\section{Analysis of an influence diagram}

In order to perform the risk assessment under a fire design situation the Bayesian causal network in Figure 1 is supplemented by decision node 13 and four utility nodes 14 , 15, 16 and 17. The purpose of the influence diagram in Figure 1 is to analyse the expected total cost $C_{t o t}$ given by equation (6). The total expected cost $C_{t o t}$ is dependent on the assumed probability of fire start $p_{f i, s}=\mathrm{P}\left(\mathrm{H}_{2}\right)$. Figure 2 shows the total cost $C_{t o t}$ as a function of the cost $C_{17}(12)$. It follows from Figure 2 that for the cost $C_{17}(12)$ up to about $5 \times 10^{5}$ (expressed in relative monetary units), the structural protection seems to be uneconomical. However for the cost $C_{17}(12)$ greater than $5 \times 10^{5}$ the expected total cost could be considerably lower when the structural protection is provided.

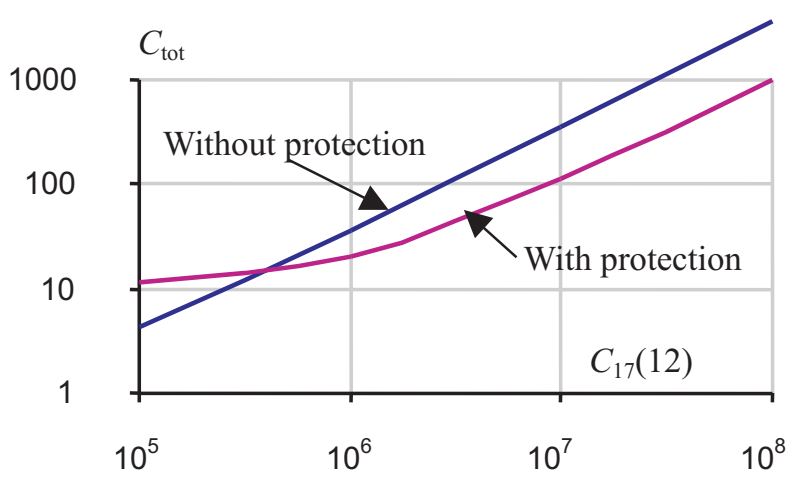

Fig. 2: Expected total cost $C_{t o t}$ versus cost $C_{17}(12)$ due to structural collapse for $p_{f i, s}=\mathrm{P}\left(H_{2}\right)=0.01$

It should be noted that the critical value of the cost $\mathrm{C}_{17}(12)$ for which the costs both with and without structural protection are equal depends on the probability of fire start $p_{f i, s}=0.01$; with increasing $p_{f i, s}$ the critical value decreases approximately by the same order.

\section{Concluding remarks}

The traditional probabilistic approach to engineering design covers only a small part of actual causes of structural failures. A significant proportion of all failures, besides gross errors, is related to hazard scenarios (e.g. fire, impact, and explosion), which are not usually included in the traditional probabilistic analysis. For this reason, specification of the design probability of failure remains an open question (how safe is safe enough?).

The methods of risk analysis and assessment are capable of encompassing more types of uncertainties than the traditional probabilistic approaches, and can significantly contribute to further improvement of advanced engineering design. The remarkable fact that the public is better prepared to accept certain risks than to stand for specified probabilities of failure will make the application of risk assessment easier. It is therefore anticipated that in the near future the probabilistic methods of structural design will be supplemented by the criteria of acceptable risks.

The above results should be considered as examples valid for the assumed input data only. These data were assessed here without due regard to specific technological and economic conditions, which should be considered in the fire safety assessment of a particular structure. Further research is needed to specify a more detailed Bayesian network and the appropriate input conditional probabilities. In particular, cost distribution depending on the states of the parent nodes should be investigated. Nevertheless, available experience indicates that the Bayesian belief network provides a very logical and effective tool for analysing the probability of fire flashover for particular fire protection conditions.

\section{Acknowledgement}

This research has been conducted at the Klokner Institute of the Czech Technical University in Prague, Czech Republic as a part of research project CEZ: J04/98/210000029 "Risk Engineering and Reliability of Technical Systems".

\section{References}

[1] Thoft-Christensen, P., Baker, M. J.: Structural Reliability and its Applications. Springer-Verlag Berlin, 1982

[2] Melchers, R. E.: Structural Reliability Analysis and Prediction. John Wiley \& Sons, Chichester, 1999

[3] Holický, M.: Fuzzy Probabilistic Optimisation of Building Performance. Automation in Construction, Elsevier, Amsterdam, 8(4), 1999, pp. 437-443.

[4] Stewart, M. G., Melchers, R. E.: Probabilistic Risk Assessment of Engineering Systems. Chapman \& Hall, London, 1997

[5] Ellingwood, B. R.: Probability-Based Structural Design: Prospect for Acceptable Risk Bases. In: Application of Statistics and Probability ICASP 8. Balkema Rotterdam, 1999, pp. 11-18

[6] Holický, M., Schleich, J.-B.: Fire Safety Assessment using Bayesian Causal Network. In: Foresight and Precaution Conference, Edinburgh, May 2000, pp. 1301-1306

[7] Holický, M., Schleich, J.-B.: Estimation of Risk under Fire Design Situation. In: Proc. of Risk Analysis 2000 Conference, Bologna, WITpress, Southampton, Boston, 2000, pp. 63-72

Doc. Ing. Milan Holický, PhD., DrSc.

phone: +420224310208

fax: +420024355232

e-mail: holicky@vc.cvut.cz

Czech Technical University in Prague

Klokner Institute

Šolínova 7, 16608 Praha 6, Czech Republic 